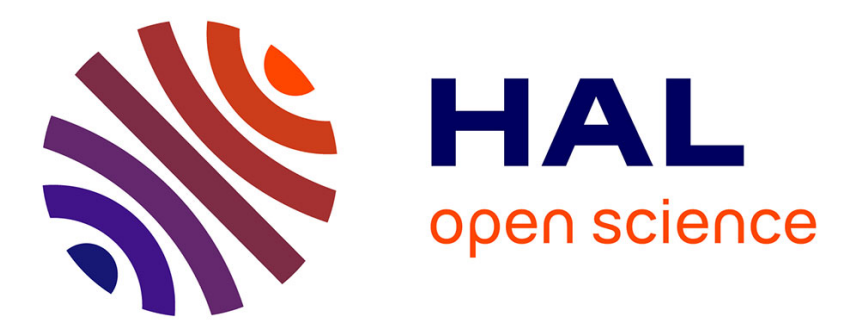

\title{
Estimation of the Distribution of Randomly Deployed Wireless Sensors
}

\author{
Merouane Debbah, Babar Khan H., T.Y. Al-Naffouri, Ryan Oyvind
}

\section{To cite this version:}

Merouane Debbah, Babar Khan H., T.Y. Al-Naffouri, Ryan Oyvind. Estimation of the Distribution of Randomly Deployed Wireless Sensors. IEEE International Symposium on Information Theory, Jun 2009, South Korea. 5 p. hal-00447065

\section{HAL Id: hal-00447065 \\ https://hal-centralesupelec.archives-ouvertes.fr/hal-00447065}

Submitted on 19 Jan 2010

HAL is a multi-disciplinary open access archive for the deposit and dissemination of scientific research documents, whether they are published or not. The documents may come from teaching and research institutions in France or abroad, or from public or private research centers.
L'archive ouverte pluridisciplinaire HAL, est destinée au dépôt et à la diffusion de documents scientifiques de niveau recherche, publiés ou non, émanant des établissements d'enseignement et de recherche français ou étrangers, des laboratoires publics ou privés. 


\section{Estimation of the Distribution of Randomly Deployed Wireless Sensors}

\author{
Babar H. Khan, \\ Department of Electrical Engineering \\ King Fahd University of Petroleum \& Minerals \\ Dhahran, 31261, K.S.A \\ Email: babar.hasan.1@gmail.com \\ Øyvind Ryan, \\ Centre of Mathematics for Applications \\ University of Oslo \\ Blindern, 0316 Oslo, Norway \\ Email: oyvindry@ifi.uio.no
}

\author{
Mérouane Debbah, \\ Alcatel-Lucent Chair, Supelec \\ Plateau de Moulon, 3 rue Joliot-Curie \\ 91192 Gif sur Yvette, France \\ Email: merouane.debbah@supelec.fr \\ Tareq Y. Al-Naffouri, \\ Department of Electrical Engineering \\ King Fahd University of Petroleum \& Minerals \\ Dhahran, 31261, K.S.A \\ Email: naffouri@kfupm.edu.sa
}

\begin{abstract}
The distribution of randomly deployed wireless sensors plays an important role in the quality of the methods used for data acquisition and signal reconstruction. Mathematically speaking, the estimation of the distribution of randomly deployed sensors can be related to computing the spectrum of Vandermonde matrices with non-uniform entries. In this paper, we use the recent free deconvolution framework to recover, in noisy environments, the asymptotic moments of the structured random Vandermonde matrices and relate these moments to the distribution of the randomly deployed sensors. Remarkably, the results are valid in the finite case using only a limited number of sensors and samples.
\end{abstract}

\section{INTRODUCTION}

Sensor networks can be used for a variety of important applications, such as measuring or monitoring temperature, sunlight or seismic activity in an area [1]. The randomness of the deployment of the sensors may have several reasons. For example, a case of practical interest is when wireless sensors are released from an airplane at fixed intervals but happen to land on earth with random positions. For a given distribution of randomly deployed sensors, one would like to infer on the distribution of the sensors. There are several cases where this is of practical interest: typically, the change of the distribution of the sensors (from a fixed pattern to a random pattern) can provide an estimate of the speed of the weed in environment monitoring. The estimation of the entire distribution can also improve signal reconstruction as recently proposed in [2]-[3]. In environment monitoring, different algorithms can be used to estimate the sensors location [4]-[5] but these algorithms require a substantial amount of communication between the sensors in large networks. In this paper, we provide a way to estimate the distribution of the sensors in noisy environments without any communication between the sensors. The results are based on the recent framework of free deconvolution [10], [6], [7] and asymptotic Vandermonde random matrix theory [8]. Interestingly, although the result are valid in the asymptotic regime, simulations using realistic random distribution deployment of sensors show the adequacy of the approach. This paper is organized as follows, Section II-A describes the system model used. Section III provides the algorithm to estimate the distribution of the sensors. Simulation results which sustain our theoretical claims are summarized in section IV followed by the conclusion.

\section{SENSOR DEPLOYMENT}

In the following, (. ${ }^{H}$ will represent the Hermitian transpose. We denote scalar quantities and matrices by lower case letters and upper case letters respectively while lower case boldface letters denote column vectors. $\operatorname{Tr}(\mathbf{A})$ represents a trace of a matrix $\mathbf{A}$ and $\operatorname{tr}_{L}(\mathbf{A})$ represents a normalized trace $\left(\frac{1}{L} \operatorname{Tr}(\mathrm{A})\right)$ of a matrix $\mathbf{A}$.

\section{A. System Model}

In the following, for simplicity sake and without loss of generality, we consider a one dimensional physical field with $L$ sensors deployed in the interval $[0,1]$. Let $d_{i} \in[0,1]$ represent the position of the $i^{\text {th }}$ sensor in the normalized interval. The continuous-time band-limited sensed signal $y\left(\omega_{i}\right)$ measured at the spatial position $\omega_{i}=2 \pi d_{i}$ can be represented as the weighted sum of $P$ harmonics,

$$
y\left(\omega_{i}\right)=\frac{1}{\sqrt{P}} \sum_{k=0}^{P-1} x_{k} e^{-j k \omega_{i}}
$$

where $i=1,2, \ldots, L . x_{k}$ is the corresponding Fourier coefficients of the $k^{\text {th }}$ harmonic. We suppose that the $L$ samples are sent from the sensors to a common data-collecting unit through an orthogonal multiple access (TDMA for example) additive Gaussian noise channel. In this case, the model can be written in a vector form as:

$$
\mathbf{y}=\mathbf{V}^{H} \mathbf{x}+\sigma \mathbf{n}
$$


where $\mathbf{y}$ is the received signal vector of length $L$ whose $i^{\text {th }}$ element is $y\left(\omega_{i}\right), \mathbf{x}$ is the transmitted signal of length $P$ whose $k^{\text {th }}$ element is $x_{k}, \mathbf{n}$ is the additive white Gaussian noise with unit variance noise vector of length $L$ whereas $\sigma^{2}$ is the noise variance. $\mathbf{V}$ is a $P \times L$ Vandermonde matrix given by,

$$
\mathbf{V}=\frac{1}{\sqrt{P}}\left(\begin{array}{lll}
1 & \cdots & 1 \\
e^{-j \omega_{1}} & \cdots & e^{-j \omega_{L}} \\
\vdots & \ddots & \vdots \\
e^{-j(P-1) \omega_{1}} & \cdots & e^{-j(P-1) \omega_{L}}
\end{array}\right)
$$

Here $\omega_{1}, \omega_{2}, \ldots, \omega_{L}$ are i.i.d random variables with a certain distribution (related to the position of the sensors) and are bounded within the interval $[0,2 \pi)$. We suppose that we have $K$ observations of the signal vector $y$. In this case, the model takes the following matrix form:

$$
\mathbf{Y}=\mathbf{V}^{H} \mathbf{X}+\sigma \mathbf{N}
$$

Where $\mathbf{Y}=\left[\mathbf{y}_{1}, \mathbf{y}_{2}, \ldots, \mathbf{y}_{K}\right], \mathbf{X}=\left[\mathbf{x}_{1}, \mathbf{x}_{2}, \ldots, \mathbf{x}_{K}\right]$ and $\mathbf{N}=$ $\left[\mathbf{n}_{1}, \mathbf{n}_{2} \ldots \mathbf{n}_{K}\right]$. The sample covariance matrix is defined as $\mathbf{Y Y}^{H}$.

In this paper, we assume that the matrix $\mathbf{X}$ of unknown transmitted symbols and the noise matrix $\mathbf{N}$ are zero mean Gaussian matrices with i.i.d. entries of unit variance. Without loss of generality, we will consider $\sigma^{2}=1$. We will define the sample covariance associated with $\mathbf{Y}$ as $\mathbf{Y} \mathbf{Y}^{H}$. Moreover, we will consider the asymptotic regime where $c_{1}=\lim _{P \rightarrow \infty} \frac{P}{K}$, $c_{2}=\lim _{P \rightarrow \infty} \frac{L}{P}$ and $c_{3}=\lim _{K \rightarrow \infty} \frac{L}{K}$. Note that although $L$ (number of sensors) and $K$ are known (number of samples), $P$ is unknown.

\section{B. Distribution Estimation}

The estimation of the distribution of $\omega$ in eq. (3) enables us to retrieve the distribution location of the sensors. In a blind context, with no training sequence and no communication between the sensors, this can be a hard task. However, as we will see afterwards, the moments of $\mathbf{V} \mathbf{V}^{H}$ can be estimated and related to the distribution of the deployed sensors by using the moments approach. In particular, we relate the moments of $\mathbf{V V}^{H}$ up to a certain order with a polynomial approximation of the distribution of $\omega$.

\section{MOMENTS APPROACH}

The moments approach [9] provides us with a good estimator of the moments of the Vandermonde matrix. The moment $\left(m_{n}\right)$ of a $P \times P$ matrix $\mathbf{H}$ is defined as

$$
m_{n}=\operatorname{tr}_{\mathrm{P}}\left(\mathbf{H}^{\mathrm{n}}\right)
$$

As recently shown in [6], [8], Free deconvolution relates the eigenvalue distribution of the covariance matrix $\left(\mu_{\mathbf{Y Y}^{H}}\right)$ with the eigenvalue distribution of the Vandermonde matrix $\left(\mu_{\mathbf{V} \mathbf{V}^{H}}\right)$ as

$$
\mu_{\mathbf{V} \mathbf{V}^{H}}=\left(\mu_{\mathbf{Y} \mathbf{Y}^{\mathbf{H}}} \boxminus_{c_{1}} \mu_{\mathbf{N N}^{H}}\right) \nabla \mu_{\mathbf{X X}^{H}}
$$

Where $\boxminus_{c_{1}}$ is Rectangular additive free deconvolution (section III-A) and $\nabla$ is multiplicative free deconvolution (section IIIB). In algorithmic terms, the moments estimation procedure follows four steps:

\section{A. Step 1: Rectangular additive free deconvolution}

Consider the covariance matrix

$$
\mathbf{Y} \mathbf{Y}^{\mathbf{H}}=(\mathbf{A}+\mathbf{N})(\mathbf{A}+\mathbf{N})^{H}
$$

where $\mathbf{A}=\mathbf{V}^{H} \mathbf{X}$. Rectangular additive free deconvolution $\left(\boxminus_{c_{1}}\right)$ provides us with the moments of $\mathbf{Y} \mathbf{Y}^{\mathbf{H}}$ in terms of moments of $\mathbf{A} \mathbf{A}^{H}$ and moments of $\mathbf{N} \mathbf{N}^{H}$. In order to compute the series of moments, it turns out that it is much easier to compute cumulants. In free probability theory, the moments $\left(m_{n}\right)$ are related to the sequence of numbers called the rectangular free cumulants $\left(t_{n}\right)$ via the probability measure $\epsilon$. [9] gives the following set of equations for the relation between the two.

$$
T_{\epsilon}\left(z\left(c_{2} M_{\epsilon^{2}}(z)+1\right)\left(M_{\epsilon^{2}}(z)+1\right)\right)=M_{\epsilon^{2}}(z)
$$

where

$$
T_{\epsilon}(z)=\sum_{n \geq 1} t_{n}(\epsilon) z^{n} \text { and } M_{\epsilon^{2}}(z)=\sum_{n \geq 1} m_{n}(\epsilon) z^{n} .
$$

This equation can be written in a recursive form as

$$
\begin{aligned}
m_{0}(\epsilon)= & 1 \\
m_{n}(\epsilon)= & t_{n}(\epsilon) \\
& +\sum_{k=1}^{n-1} c_{1}^{k} t_{k}(\epsilon) \sum_{\substack{l_{1}, \ldots, l_{2 k} \geq 0 \\
l_{1}+\ldots+l_{2 k}=n-k}} m_{l_{1}}(\epsilon) \ldots m_{l_{2 k}}(\epsilon)
\end{aligned}
$$

Let $\gamma, \eta$ and $\tau$ be the probability measure of $\mathbf{Y} \mathbf{Y}^{H}, \mathbf{A} \mathbf{A}^{H}$ and $\mathbf{N N}^{H}$ respectively. In this case, these are related by [10]:

$$
t_{n}(\eta)=t_{n}(\gamma)-t_{n}(\tau)
$$

Note that as $\mathbf{N}$ is a random matrix with independent Gaussian entries with variance $\frac{1}{L}$ then the eigenvalue distribution of $\mathbf{N N}^{H}$ follow a Marchenko-Pastur distribution with parameter $\frac{1}{c_{3}}$. In this case the rectangular free cumulants of $\mathbf{N N}^{H}$ are given by [10] $t_{n}(\tau)=\delta_{n, 1}{ }^{1}, \forall n \geq 1$.

Hence, the rectangular additive free deconvolution provides us with the moments of $\mathbf{V}^{H} \mathbf{X} \mathbf{X}^{H} \mathbf{V}$.

\section{B. Step 2: Multiplicative free deconvolution}

In this section, we show how one can extract the moments of $\mathbf{V} \mathbf{V}^{H}$ from $\mathbf{V}^{H} \mathbf{X} \mathbf{X}^{H} \mathbf{V}$. As a first step, note that:

$$
m_{n}\left(\mathbf{X} \mathbf{X}^{\mathbf{H}} \mathbf{V} \mathbf{V}^{H}\right)=c_{2} m_{n}\left(\mathbf{V}^{H} \mathbf{X} \mathbf{X}^{H} \mathbf{V}\right)
$$

We can therefore use the concept of Multiplicative free deconvolution $(\nabla)$ which computes the moments of $\mathbf{V} \mathbf{V}^{H}$ in

\footnotetext{
${ }^{1}$ The dirac delta function is defined as,

$$
\delta_{n, 1}= \begin{cases}1 & \text { if } n=1 \\ 0 & \text { else }\end{cases}
$$
}


terms of the moments of $\mathbf{X} \mathbf{X}^{H} \mathbf{V} \mathbf{V}^{H}$ and moments of $\mathbf{X} \mathbf{X}^{H}$. As previously, from an algorithmic perspective, it is easier to compute cumulants. The relationship between the moments $m_{n}$ and the multiplicative free cumulants $\left(s_{n}\right)$ is given by [8]:

$$
M_{\epsilon}(z) S_{\epsilon}\left(M_{\epsilon}(z)\right)=z\left(1+M_{\epsilon}(z)\right)
$$

where,

$$
S_{\epsilon}(z)=\sum_{n \geq 1} s_{n}(\epsilon) z^{n-1} \text { and } M_{\epsilon}(z)=\sum_{n \geq 1} m_{n}(\epsilon) z^{n} .
$$

These set of equations can be represented in a recursive form as

$$
\begin{aligned}
& m_{1}(\epsilon) s_{1}(\epsilon)=1 \\
& m_{n}(\epsilon)=\sum_{k=1}^{n+1} s_{k}(\epsilon) \sum_{\begin{array}{c}
l_{1}, \ldots, l_{k} \geq 1 \\
l_{1}+\ldots+l_{k}=n+1
\end{array}} m_{l_{1}}(\epsilon) \ldots m_{l_{k}}(\epsilon)
\end{aligned}
$$

Let $\vartheta, \varsigma$ and $\psi$ be the probability measures of $\mathbf{X} \mathbf{X}^{H} \mathbf{V} \mathbf{V}^{H}$, $\mathbf{X X}^{H}$ and $\mathbf{V} \mathbf{V}^{H}$ respectively. Then these probability measures are related to each other thought the multiplicative free cumulants as

$$
\begin{aligned}
s_{1}(\varsigma) s_{1}(\psi)= & s_{1}(\vartheta) \\
s_{1}(\varsigma) s_{n}(\psi)= & s_{n}(\vartheta)-s_{n}(\varsigma) s_{1}(\psi) \\
& -\sum_{k=2}^{n-1} s_{k}(\varsigma) s_{n+1-k}(\psi)
\end{aligned}
$$

Note that if $\mathbf{X}$ is a $P \times K$ random matrix with independent Gaussian entries with variance $\frac{1}{P}$ then the eigenvalue distribution of $\mathbf{X X}^{H}$ follow a Marchenko-Pastur distribution with parameter $\frac{1}{c_{1}}$. In this case the multiplicative free cumulants of $\mathbf{X X}^{H}$ are given by $s_{n}(\varsigma)=\left(-c_{1}\right)^{n-1}, \forall n \geq 1$.

\section{Step 3: Moments of $\mathbf{V} \mathbf{V}^{H}$}

In the following, we assume that $\omega_{i}=2 \pi(i-1)+\omega_{i}^{\prime}$ where $i=1,2, \ldots, L$. In other words, all the sensors are centered at equally spaced positions with a certain off-set. Here $\omega_{i}^{\prime}$ is a random variable with continuous (not necessarily uniform) distribution and is bounded by $[0,2 \pi)$. We suppose that all $\omega_{i}^{\prime}$ $, i=1,2, \ldots, L$ have the same distribution.

The asymptotic moments of the Vandermonde matrix are defined as

$$
m_{n}=\lim _{P \rightarrow \infty} E\left[\operatorname{tr}_{\mathrm{P}}\left(\mathbf{V} \mathbf{V}^{\mathrm{H}}\right)^{\mathrm{n}}\right]
$$

It has been shown recently in [8] that for any distribution of the random phases, the moments of the Vandermonde matrix can be calculated as

$$
\sum_{\rho \in \mathcal{P}(n)} K_{\rho, \omega} c_{2}^{|\rho|}
$$

where $\mathcal{P}(n)$ is the set of all partitions of $\{1,2, \ldots, n\}$ and $\rho$ is the notation for a particular partition in $\mathcal{P}(n)$. This can be also written as $\rho=\left\{\rho_{1}, \ldots, \rho_{k}\right\}$, where $\rho_{j}$ are the blocks of $\rho$ and $|\rho|$ is the number of blocks in $\rho . K_{\rho, \omega}$ are called the
Vandermonde mixed moment expansion coefficient and are defined, in the case where $\omega$ is a uniform distribution $\omega \sim$ $\mathcal{U}(0,1)^{2}$ as

$$
\begin{aligned}
K_{\rho, \omega}= & \lim _{N \rightarrow \infty} \frac{1}{P^{n+1-|\rho|}} \\
& \int_{(0,2 \pi)^{|\rho|}} \prod_{k=1}^{n} \frac{1-e^{j P\left(\omega_{b(k-1)}-\omega_{b(k)}\right)}}{1-e^{j P\left(\omega_{b(k-1)}-\omega_{b(k)}\right)}} d \omega_{1} \ldots d \omega_{|\rho|}
\end{aligned}
$$

Interestingly, the moments of the Vandermonde matrix can be written in terms of the distribution of $p_{\omega}$ of $\omega$ as

$$
m_{n}=\sum_{\rho \in \mathcal{P}(n)} K_{\rho, u} c_{2}^{|\rho|} I_{|\rho|}
$$

where $I_{|\rho|}=(2 \pi)^{|\rho|-1}\left(\int_{0}^{2 \pi} p_{\omega}(x)^{|\rho|} d x\right)$ and $u \sim \mathcal{U}(0,1)$. In general, it is extremely difficult to obtain an explicit expression of $K_{\rho, u}$ for any moments (in [8] only the first seven moments were computed). In this paper, we provide an algorithm to calculate all the moments:

Algorithm: $K_{\rho, u}$ can be expressed as the volume of the solution set of

$$
\sum_{k \in \rho_{j}} l_{k-1}=\sum_{k \in \rho_{j}} l_{k}
$$

with $0 \leq l_{k} \leq 1$. This volume is calculated after expressing $|\rho|-1$ variables in terms of $n+1-|\rho|$ free variables and is bounded within $[0,1]$. Note that $K_{\rho, u}=1$ when the partitions of $\rho$ are non-crossing [11] otherwise it is smaller the 1. As $I_{|\rho|}$ depends on the block cardinalities $\left|\rho_{j}\right|$, we can therefore group together $K_{\rho, u}$ for $\rho$ with equal block cardinalities. Hence, we group the cardinalities in descending order $r_{1} \geq r_{2} \geq \ldots \geq r_{k}$ and define

$$
K_{r_{1}, r_{2}, \ldots, r_{k}}=\sum_{\hat{\rho} \in \mathcal{P}(n)} K_{\rho, u}
$$

where $\left|\hat{\rho}_{i}\right|=r_{i}, \forall i$. In this case, the moments can be represented as

$$
m_{n}=\sum_{k=1,2, \ldots, n} \sum_{\begin{array}{c}
r_{1}, r_{2}, \ldots, r_{k} \\
r_{1}+r_{2}+\cdots+r_{k}=n
\end{array}} K_{r_{1}, r_{2}, \ldots, r_{k}} c_{2}^{k} I_{k}
$$

As an example, the first moments expand to:

$$
\begin{aligned}
m_{1}= & K_{1} c_{2} I_{1} \\
m_{2}= & K_{2} c_{2} I_{1}+K_{1,1} c_{2}^{2} I_{2} \\
m_{3}= & K_{3} c_{2} I_{1}+K_{2,1} c_{2}^{2} I_{2}+K_{1,1,1} c_{2}^{3} I_{3} \\
m_{4}= & K_{4} c_{2} I_{1}+\left(K_{3,1}+K_{2,2}\right) c_{2}^{2} I_{2}+K_{2,1,1} c_{2}^{3} I_{3} \\
& +K_{1,1,1,1} c_{2}^{4} I_{4} \\
\vdots & \vdots
\end{aligned}
$$

\footnotetext{
${ }^{2}$ here $U$ is the uniform distribution.
} 


\section{Step 4: distribution Approximation}

Now in order to estimate the distribution of $\omega$, where $\omega$ is bounded within $[0,1)$, we use the Weierstrass approximation and multinomial expansion to derive an alternative form of $I_{n}$ given by

$$
\begin{aligned}
I_{n}= & \lim _{t \rightarrow \infty}(2 \pi)^{n-1} \sum_{k_{1}+\ldots+k_{t}=n}\left(\begin{array}{c}
n \\
k_{1}, \ldots, k_{t}
\end{array}\right) \\
& \cdot\left(\frac{\Gamma\left(1+t n-\sum_{v=1}^{t} v k_{v}\right) \Gamma\left(\sum_{v=1}^{t} v k_{v}-n+1\right)}{\Gamma((t-1) n+2)}\right) \\
& \cdot\left(\prod_{v=1}^{t}\left[p_{\omega}\left(\frac{v-1}{t-1}\right)\left(\begin{array}{c}
t-1 \\
v-1
\end{array}\right)\right]^{k_{v}}\right)
\end{aligned}
$$

where $p_{\omega}(x)$ represents the unknown distribution of $\omega$ and $p_{\omega}(0), p_{\omega}\left(\frac{1}{t-1}\right), p_{\omega}\left(\frac{2}{t-1}\right), \ldots, p_{\omega}(1)$ are the unknowns of the weierstrass approximation. By equating (17) with (16), this will give us a set of non-linear equations to solve. As the number of unknowns should be equal to the number of equations, we take $n=t$. One can solve this by using any optimization algorithm.

\section{Simulation Results}

In this section, we provide some simulation results to sustain our theoretical claims. We apply the previous four step procedure for various values of $K, L$ and $P$. We restrict also our analysis to some specific distribution in order to assess the validity of our results in various case.

\section{A. Estimation of the moments of $\mathbf{V} \mathbf{V}^{H}$}

By applying rectangular additive free deconvolution and multiplicative free deconvolution on the covariance matrix $\mathbf{Y} \mathbf{Y}^{H}$, we can compute the moments of $\mathbf{V} \mathbf{V}^{H}$. To check the validity of the moments approach, we plot the relative distance between the estimated moments of $\mathbf{V} \mathbf{V}^{H}$ and the actual moments of $\mathbf{V} \mathbf{V}^{H}$. The relative distance we use is defined as, $\forall n$ :

$$
\left|\frac{m_{n}\left[\left(\mu_{\mathbf{Y Y}}{ }^{H} \boxminus_{c_{1}} \mu_{\mathbf{N N}^{H}}\right) \nabla \mu_{\mathbf{X X}^{H}}\right]}{m_{n}\left[\mathbf{V} \mathbf{V}^{H}\right]}-1\right|
$$

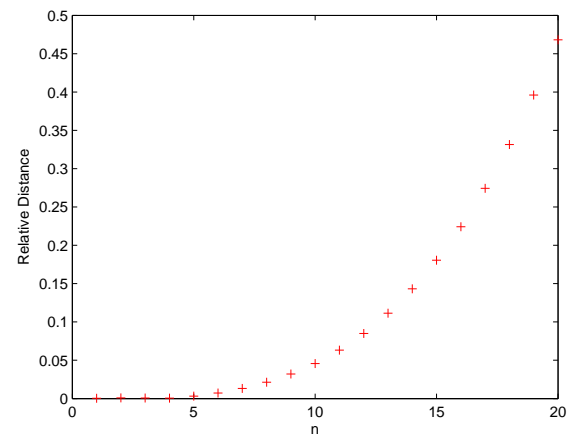

Fig. 1. Relative distance of the moments of $\mathbf{V} \mathbf{V}^{H}$ and the estimated moments by applying the moments approach with $K=L=P=1000$.
Figure 1 plots the relative distance for $n=1,2, \ldots, 20$ ( $n$ is the order of the moments) with $K=L=P=1000$ when $\omega$ with a uniform distribution. Interestingly, even with only $K=1000$ samples, the error is quite low for moments inferior to 10. If one requires higher moments, one would need more moments.

\section{B. Von Mises Distribution}

Let us consider the case where $\omega$ has a Von Mises distribution. Von Mises distribution have an inverted bell shape and are bounded within $(0,2 \pi]$. The parameter $1 / \kappa$ of the distribution is analogous to $\sigma$ in a Gaussian distribution. The value of $I_{2}$ for a von mises distribution is given by

$$
I_{2}=\frac{J_{0}(2 \kappa)}{J_{0}(\kappa)^{2}}
$$

where $J_{0}$ is the bessel function of the zero order.

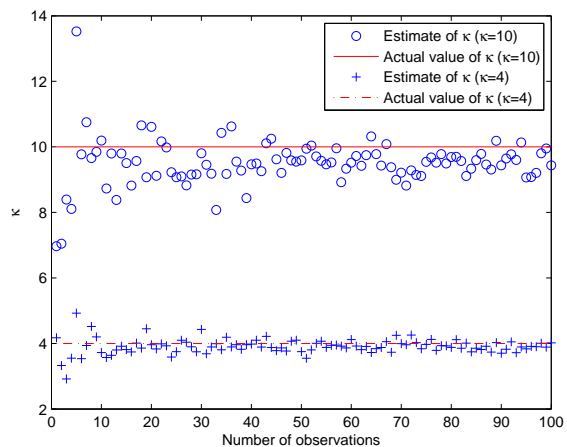

Fig. 2. Estimation of $\kappa$ for Von Mises distribution with $K=L=P=100$.

The value of $\kappa$ is estimated by solving equation (16) and (18). Figure 2 shows the simulation result for $\kappa=4$ and $\kappa=10$ with $K=L=P=100$ averaged over a varying number of observations. Figure 3 simulates the result for the estimation of $\kappa$ as $K, L$ and $P$ increase linearly. It can be seen that by asymptotically increasing the value of $K, L$ and $P$ the estimation gets better.

\section{Distribution Approximation}

We consider now the general case where there is no constraint on the distribution of $\omega$ except that it is bounded in the interval $[0,1]$. For simulations purposes, we consider $\omega$ to have a beta distribution with two degrees of freedom $\alpha$ and $\beta$. The PDF is given by,

$$
p_{\omega}(x)=\frac{x^{\alpha}(1-x)^{\beta}}{B(\alpha, \beta)}
$$

By taking $n=t=9$ in (17) with (16), we obtain a a set of non-linear equations of order 9 . Here we use nonlinear leastsquares based algorithm [12] to calculate the unknowns.

Figure 4 shows the simulation result for the estimation of the distribution of $\omega$ with $t=3,5,9$ when $\omega$ has beta distribution with $\alpha=2$ and $\beta=5$. Figure 5 represents the estimation of the PDF when $\omega$ has beta distribution with $\alpha=1, \beta=3$ and 


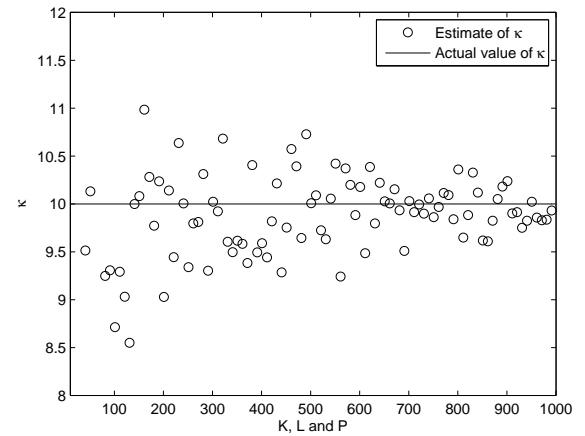

Fig. 3. Estimation of $\kappa$ for Von Mises distribution with varying $K, L$ and $P$.

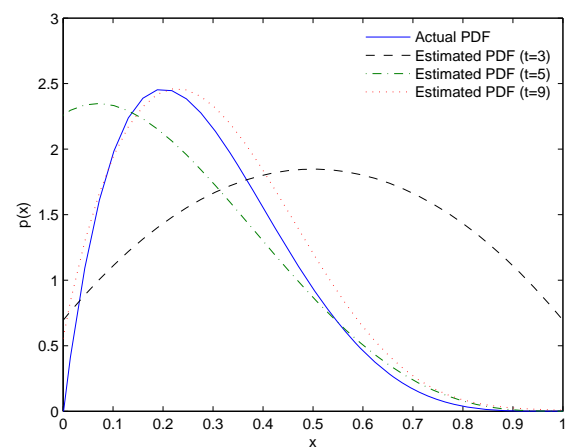

Fig. 4. Estimation of the PDF of $\omega$, where $\omega$ has a beta distribution with $\alpha=2$ and $\beta=5$.

$t=3,5,9$. Similarly, Figure 6 shows the estimate of the beta distribution with $\alpha=1, \beta=3$ and $t=3,5$. In each case, with more than 5 moments we already get a good approximation of the distribution. Note that we can loosen the bound on the range of $\omega$ from $[0,1)$ to $[0,2 \pi)$ by replacing $t$ by $t / 2 \pi$ in (17).

\section{CONCLUSION}

In this paper, we showed that by using the moments approach, we can find an estimate for the distribution of the sensors with very few noisy observations and without any communication between the sensors. This is very helpful for large networks of randomly deployed sensors where one needs to recover the distribution.

\section{REFERENCES}

[1] W. S. Conner, J. Heidemann, L. Krishnamurthy, X. Wang, and M. Yarvis, "Workplace Applications of Sensor Networks," Technical Report ISI-TR2004-591, USC/Information Sciences Institute, July, 2004.

[2] A. Nordio, C.F. Chiasserini and E. Viterbo, "Bandlimited Field Reconstruction for Wireless Sensor Networks," http:/arxiv.org/PS_cache/arxiv/pdf/0707/0707.1954v1.pdf

[3] P. Marziliano, M. Vetterli, "Reconstruction of Irregularly Sampled Discrete-Time Bandlimited Signals with Unknown Sampling Locations," IEEE Trans. on Signal Processing, vol. 48, no.12, pp. 3462-3471, Dec. 2000.

[4] L. Hu, D. Evans, "Localization for Mobile Sensor Networks," Tenth Annual International Conference on Mobile Computing and Networking (ACM MobiCom 2004), Philadelphia, PA, September-October 2004.

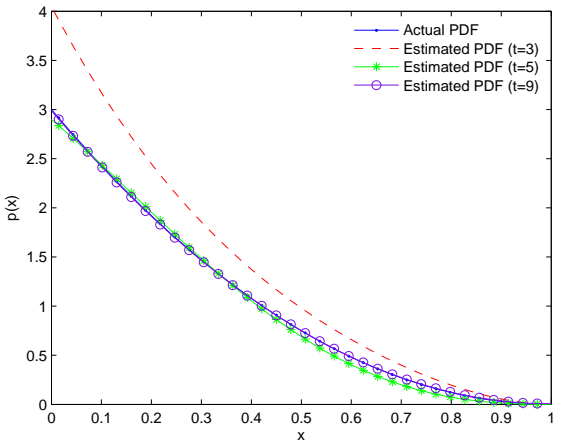

Fig. 5. Estimation of the PDF of $\omega$, where $\omega$ has a beta distribution with $\alpha=1$ and $\beta=3$.

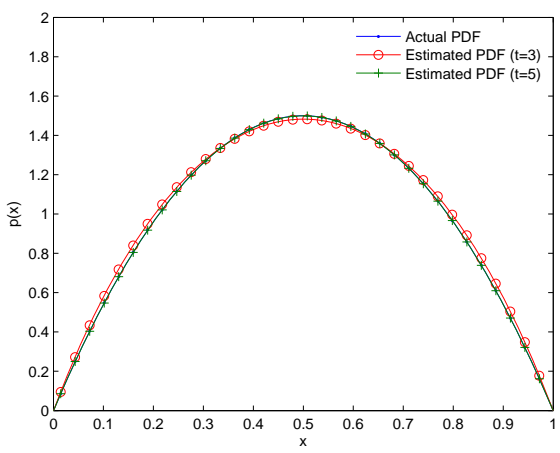

Fig. 6. Estimation of the PDF of $\omega$, where $\omega$ has a beta distribution with $\alpha=2$ and $\beta=2$.

[5] O. Kwon, H. Song, "Localization through Map Stitching in Wireless Sensor Networks," IEEE Transactions on Parallel and Distributed Systems, vol. 19, no. 1, pp. 93-105, Jan. 2008.

[6] Ø. Ryan and M. Debbah "Free Deconvolution for Signal Processing Applications," IEEE International Symposium on Information Theory, ISIT 2007, June 2007.

[7] Ø. Ryan and M. Debbah "Channel Capacity Estimation using Free Probability theory," IEEE Trans. Signal Process., Vol. 56, No. 11, 2008, pp. 5654-5667.

[8] Ø. Ryan and M. Debbah "Asymptotic Behaviour of Random Vandermonde Matrices with Entries on the Unit Circle," to appear in IEEE Trans. on Information Theory, 2009.

[9] F. Benaych-Georges and M. Debbah "Free Deconvolution: from Theory to Practice," submitted to IEEE Trans. on Information Theory, 2008

[10] F. Benaych-Georges "Infinitely divisible distribution for rectangular free convolution: classification and matricial interpretation," Probab. Theory Related Fields, vol. 139, no. 1-2, pp. 143-189, 2007.

[11] R. Speicher "Free Probability Theory and Non-Crossing partitions," Séminaire Lotharingien de Combinatoire, B39c (1997), 38 pages, 1997.

[12] Coleman, T.F. and Y. Li "An Interior, Trust Region Approach for Nonlinear Minimization Subject to Bounds," SIAM Journal on Optimization, vol. 6, pp. 418-445, 1996. 